\title{
Disk degeneration of the upper lumbar disks is associated with hip pain
}

\author{
Evelien I. T. de Schepper · Jurgen Damen • \\ Pieter K. Bos • Albert Hofman • Bart W. Koes • \\ Sita M. Bierma-Zeinstra
}

Received: 28 June 2011/Revised: 7 August 2012/Accepted: 28 October 2012/Published online: 8 November 2012

(c) Springer-Verlag Berlin Heidelberg 2012

\begin{abstract}
Purpose A possible cause of hip pain is the presence of radiating pain from the higher lumbar spine. Identification of factors associated with hip pain arising from the lumbar spine would aid the physician. The first step in identifying possible factors is to look at the association between hip pain and osteoarthritis of the lumbar spine.

Methods In an open population based study of people 55 years and older (Rotterdam study), 2,819 lumbar radiographs were scored for the presence and severity of individual radiographic features of disk degeneration. Hip osteoarthritis was scored on anteroposterior pelvic radiographs, and questionnaires including self-reported hip pain were taken. Logistic regression adjusted for possible confounders was used to determine the association between self-reported hip pain and the individual radiographic features of lumbar disk degeneration.

Results The presence of dis space narrowing grade $\geq 1$ at level L1/L2 was significantly associated with hip pain in the last month (men $\mathrm{OR}=2.0 ; 95 \%$ CI $1.1-3.8$ and women $\mathrm{OR}=1.7 ; 95 \%$ CI 1.1-2.5). The presence of disk space narrowing grade $\geq 1$ at level L2/L3 was only significantly associated with hip pain in women. The strength
\end{abstract}

E. I. T. de Schepper $(\bowtie) \cdot$ J. Damen · B. W. Koes ·

S. M. Bierma-Zeinstra

Department of General Practice, Erasmus MC, PO Box 2040, 3000 CA Rotterdam, The Netherlands

e-mail: e.deschepper@erasmusmc.nl

P. K. Bos

Department of Orthopedics, Erasmus MC, PO Box 2040, 3000 CA Rotterdam, The Netherlands

A. Hofman

Department of Epidemiology, Erasmus MC, PO Box 2040,

3000 CA Rotterdam, The Netherlands of the associations increased for self-reported chronic hip pain, especially in men (L1/L2 OR $=2.5 ; 95 \% \mathrm{CI}$ 1.3-5.0). The presence of disk space narrowing at the lower levels (L3/L4/L5/S1) was not significantly associated with hip pain.

Conclusion Our data provide evidence for an association between hip pain and disk space narrowing at disk level L1/L2 and L2/L3. In case of uncertainty of the cause of hip pain, evaluation of lumbar radiographs may help to identify those hip pain patients who might have pain arising from the lumbar spine.

Keywords Hip pain - Back pain .

Lumbar disk degeneration

\section{Introduction}

Hip pain is a common symptom among older adults, with a point prevalence of $14.3 \%$ reported in the United States [1]. The differential diagnosis of hip pain is broad and includes intra-articular pathology, extra-articular pathology, and other causes like radiating pain from the lumbar spine. Differentiating back pain from hip pain in patients who present with classic signs and symptoms is mostly not difficult and generally does not require further testing to establish an accurate diagnosis. However, in some cases, patients present with nonspecific complaints of pain in the lumbar spine, buttock, lateral hip, or thigh [2]. The differentiation of signs and symptoms suggestive of hip disorders versus spine disorders is important in giving patients the most beneficial treatment, especially if the treatment includes a major reconstructive surgery, such as hip replacement.

Differentiating whether hip pain originates from the hip, the spine, or both may be challenging. Brown et al. [3] 
attempted to determine which physical signs and symptoms best predict the primary source of pain in patients with hip-, spine-, or concomitant disorders. After final diagnosis with imaging studies, they found that although limited internal rotation, groin pain, and a limp are more commonly associated with a hip disorder, these symptoms are also seen in patients with spine alone or both hip and spine disorders.

In order to make a differentiation between hip and spine originated hip pain there have been a few studies about the usefulness of local anesthetic with(out) corticosteroid hip infiltrations, to differentiate intra-articular causes of hip pain from spinal causes [4-7]. To our knowledge, there have been no studies about the usefulness of local spine infiltrations to differentiate hip and spine originated hip pain. However, infiltration of every patient with atypical hip pain for possible coexistent lumbar spine osteoarthritis would be counterproductive and costly. Preoperative identification of factors associated with hip pain arising from the lumbar spine would aid the physician by identifying the subgroup of patients who might not experience full relief of pain with a hip arthroplasty.

One of the first steps to identify possible factors is to look at the association between hip pain and osteoarthritis of the lumbar spine. The purpose of this study was to explore the association of self-reported hip pain with the different individual radiographic features (IRF) of spinal osteoarthritis by vertebral level, including osteophytes and disk space narrowing.

\section{Materials and methods}

\section{Study population}

The data for this study originate from data of the Rotterdam Study, an open population prospective cohort of people aged 55 years and older. The objective of the Rotterdam Study is to investigate the incidence of, and risk factors for, chronic disabling diseases. The study design has been described previously [8]. All 10,275 inhabitants of Ommoord (a district in Rotterdam, the Netherlands) were invited to participate. The baseline measurements were conducted between 1990 and 1993. In total, 7,983 participants were examined.

For this study, 2,819 lumbar radiographs were scored. The selection was based on the availability of radiographs of the hip and spine at a follow-up measurement 6.6 years later $[9,12]$.

\section{Radiographic scoring}

Lumbar lateral radiographs were scored by a single observer trained by a radiologist for the presence of the individual radiographic features of disk degeneration. The observer was blinded to clinical characteristics of the participants. Each vertebral level from L1/2 to L5/S1 was reviewed for the presence and severity of osteophytes (anterior) and vertebral narrowing, using the Lane atlas $[10,11]$. In this atlas grade $0=$ none; grade $1=$ mild; grade $2=$ moderate; and grade $3=$ severe. The lumbosacral disk space was defined as narrowed when its height was less than that of the disk space between the third and fourth lumbar vertebrae. This is due to a normal progression of increasing disk space height from the third and fourth to the fourth and fifth lumbar vertebrae, and then a relative narrowing of the height of the lumbosacral disk space. Sclerosis was not scored because of the earlier reported low ICC for this feature [11].

Inter-observer reproducibility was assessed by a second independent observer who evaluated a random selection of $140(5 \%)$ X-rays. The ICC was 0.83 for osteophytes and 0.77 for vertebral narrowing, indicating good reproducibility.

Weight bearing anteroposterior radiographs of the pelvis were obtained. One trained reader evaluated the radiographs obtained at baseline, unaware of the clinical status of the participants [9]. At baseline, radiological osteoarthritis of the hip was quantified by measurements following the Kellgren \& Lawrence grading system (atlas-based) in five grades (from 0 to 4 ). A person was considered to have osteoarthritis of the hip, if the Kellgren \& Lawrence score of one or both joints was equal to or larger than two [9].

Hip pain

Hip pain and low back pain were determined from interviewing the participants during the home visits. Participants were asked "Did you have complaints of the (left and/or right) hip during the last month?" Hip pain was defined to be present if the answer was positive. Participants were subsequently asked "What is the duration of the present hip complaints?" For low back pain similar questions were asked. We defined chronic hip pain to be present if the duration of the hip joint pain was more than 1 year.

Participants also visited the research center, where X-rays were obtained. Height and weight were measured with participants wearing indoor clothing and without shoes. Body mass index (BMI) was calculated as weight in kilograms divided by length in meters squared $\left(\mathrm{kg} / \mathrm{m}^{2}\right)$.

Statistical analyses

We defined disk space narrowing to be present if the grade was mild, moderate, or severe (grade $\geq 1$ ). Because of the small proportion of subjects without osteophytes, we used a higher cutoff value for this feature. We defined osteophytes 
to be present if the grade was moderate or severe (grade 22) [12]. Using these definitions we calculated the prevalence of the IRF by vertebral level (L1/2 to L5/S1) and gender.

In order to explore the association between the IRF by vertebral level and hip pain, hip pain was used as the dependent variable with adjustments made for age and gender. The assessments of the associations were also adjusted for radiological hip osteoarthritis, as this variable was shown to be associated with disk space narrowing and of course with hip pain. The same was true for low back pain [12]. In addition, the assessments of the association were also adjusted for BMI, as this variable has been reported to be associated with both hip pain and some of the individual radiographic features [13-15].

In a separate analysis we explored the association between the IRF by vertebral level and hip pain in subjects with no sign of radiological hip osteoarthritis. The results of the analyses are expressed as odds ratios (OR) with $95 \%$ confidence intervals (CI), stratified for gender. The same methods were used to explore the association between the IRF and chronic hip pain. Statistical analysis was performed using SPSS version 15 (SPSS Inc, Chicago, USA).

\section{Results}

\section{Subject characteristics}

Baseline characteristics are shown in Table 1. There were 1,204 men [mean age 65.3 years, standard deviation (SD) 6.4] and 1,615 women (mean age 65.9 years, SD 6.8). Hip pain during the last month was reported more often by women than men [244 $(15.1 \%)$ vs. $84(7.0 \%) p<0.05$ ] (Table 1). Chronic hip pain was reported in the majority (82\%) of the current hip pain cases and was also more often reported by women [208 $(12.9 \%)$ vs. $62(5.1 \%)$ $p<0.05]$.

Radiological hip osteoarthritis was observed in 209 (7.4\%) persons (Kellgren \& Lawrence $\geq 2$ in one or both hips).

\section{Influence of gender and vertebral level}

The prevalence of the IRF in men and women is shown in Table 1. Osteophytes were the most frequently observed radiographic feature and were slightly more common in men than women (95 vs. $91 \% ; p<0.05$ ). Disk space narrowing was more frequent in women than men (65 vs. $53 \% ; p<0.05)$. In terms of their distribution by vertebral level, narrowing was more frequent at the lower lumbar disk levels.
Disk space narrowing grade $\geq 1$ at level L1/L2 was more common in persons with hip pain (19 vs. $10 \%$; $p<0.05)$. Hip pain was more common in persons with disk space narrowing grade $\geq 1$ at level L1/L2 (21 vs. $11 \% ; p<0.05$ ).

\section{Association with LDD}

Table 2 shows the association between hip pain and the IRF, adjusted for age, gender, BMI, hip arthritis, and low back pain. The presence of disk space narrowing grade $\geq 1$ at level L1/L2 was significantly associated with hip pain in the last month, both in men and women (men $\mathrm{OR}=2.0$; $95 \%$ CI $1.1-3.8$ and women OR $=1.7 ; 95 \%$ CI $1.1-2.5$ ) (Table 2). The presence of disk space narrowing grade $\geq 1$ at level L2/L3 was significantly associated with hip pain in the last month, only in women $(\mathrm{OR}=1.6 ; 95 \% \mathrm{CI}$ 1.1-2.2). The strength of the associations increased for the participants with chronic hip pain, especially for men (L1/L2 OR $=2.5 ; 95 \%$ CI 1.3-5.0). The strength of the associations also increased for the group of subjects with no radiological hip osteoarthritis (men chronic pain L1/L2 $\mathrm{OR}=2.7 ; 95 \%$ CI $1.3-5.5$ and women chronic pain $\mathrm{L} 1 / \mathrm{L} 2 \mathrm{OR}=2.0 ; 95 \%$ CI 1.3-3.2).

The presence of disk space narrowing at the lower levels (L3/L4/L5/S1) was not significantly associated with hip pain. The presence of disk space narrowing grade $\geq 2$ was not explored, because of the low number of persons with disk space narrowing grade $\geq 2$ at the upper levels.

The presence of osteophytes grade $\geq 2$ was not significantly associated with hip pain at any level (data not shown).

\section{Discussion}

The differentiation of signs and symptoms suggestive of hip disorders versus spine disorders is important in giving patients the most beneficial treatment. The purpose of this study was to explore the association of self-reported hip pain with the different individual radiographic features (IRF) of spinal osteoarthritis. In this study, disk space narrowing at level L1/L2 appeared to be associated with pain in the hip region, especially in men. The strength of the associations increased for participants with chronic hip pain and in those without radiological signs of hip osteoarthritis. These results suggest that in case of uncertainty of the cause of hip pain, evaluation of lumbar radiographs may help to identify those hip pain patients who may benefit the most from further diagnostic evaluation.

Our data provide evidence for radiating pain from the higher lumbar spine as a possible cause of hip pain in a cross-sectional open population based study. One of the explanations that can be found for the association between hip pain and disk space narrowing at level L1/L2 and 
Table 1 Frequency of hip pain and individual radiographic features of the low back in men and women

\begin{tabular}{|c|c|c|c|c|}
\hline & Men, $N=1,204$ & Women, $N=1,615$ & All, $N=2,819$ & Hip pain, $N=328$ \\
\hline Age (years) Mean \pm SD & $65.3 \pm 6.4$ & $65.9 \pm 6.8$ & $65.7 \pm 6.6$ & $66.2 \pm 6.8$ \\
\hline Body mass index (BMI) Mean $\pm \mathrm{SD}$ & $25.9 \pm 2.9$ & $26.6 \pm 3.8$ & $26.3 \pm 3.5$ & $27.0 \pm 3.9$ \\
\hline Hip pain $(\%)^{\mathrm{a}}$ & $84(7.0)$ & $244(15.1)$ & $328(11.6)$ & $328(100)$ \\
\hline Chronic hip pain $(\%)^{\mathrm{b}}$ & $62(5.1)$ & $208(12.9)$ & $270(9.6)$ & $270(82.3)$ \\
\hline Hip osteoarthritis (\%) & $94(7.8)$ & $115(7.1)$ & $209(7.4)$ & $51(15.5)$ \\
\hline \multicolumn{5}{|l|}{ Osteophytes low back (\%) } \\
\hline Grade $\geq 1$ & $1,148(95.3)$ & $1,467(90.8)$ & $2,615(92.8)$ & $306(93.3)$ \\
\hline Grade $\geq 2$ & $832(69.1)$ & $929(57.5)$ & $1,761(62.5)$ & $217(66.2)$ \\
\hline Grade 3 & $536(44.5)$ & $505(31.3)$ & $1,041(36.9)$ & $134(40.9)$ \\
\hline \multicolumn{5}{|l|}{ Narrowing low back (\%) } \\
\hline Grade $\geq 1$ & $637(52.9)$ & $1,048(64.9)$ & $1,685(59.8)$ & $210(64.0)$ \\
\hline Grade $\geq 2$ & $286(23.8)$ & $525(32.5)$ & $811(28.8)$ & $115(35.1)$ \\
\hline Grade 3 & $40(3.3)$ & $107(6.6)$ & $147(5.2)$ & $20(6.1)$ \\
\hline \multicolumn{5}{|l|}{ Osteophytes $\geq 2(\%)$} \\
\hline $\mathrm{L} 1-2$ & $282(23.4)$ & $297(18.4)$ & $579(20.5)$ & $84(25.6)$ \\
\hline L2-3 & $347(28.8)$ & $404(25.0)$ & 751 (26.6) & $105(32.0)$ \\
\hline L3-4 & $428(35.5)$ & $364(22.6)$ & $792(28.1)$ & $100(30.5)$ \\
\hline L4-5 & $403(33.5)$ & 354 (21.9) & 757 (26.9) & $94(28.7)$ \\
\hline L5-S1 & $312(25.9)$ & $303(18.8)$ & $615(21.8)$ & $68(20.7)$ \\
\hline \multicolumn{5}{|l|}{ Narrowing $\geq 1(\%)$} \\
\hline L1-2 & $107(8.9)$ & $201(12.5)$ & $308(10.9)$ & $63(19.2)$ \\
\hline L2-3 & $135(11.3)$ & $307(19.0)$ & $442(15.7)$ & $81(24.7)$ \\
\hline L3-4 & $153(12.7)$ & $342(21.1)$ & 495 (17.6) & $78(23.8)$ \\
\hline L4-5 & $268(22.2)$ & $526(32.6)$ & $794(28.2)$ & $111(33.8)$ \\
\hline L5-S1 & $408(34.0)$ & $662(41.0)$ & $1,070(38.0)$ & $127(38.7)$ \\
\hline
\end{tabular}

${ }^{a}$ Hip pain: complaints of the hip joint during last month

b Chronic hip pain: duration present hip joint complaints $>1$ year

L2/L3 is "referred pain." The term "referred pain" is used for pain localized not in the site of its origin but in areas that may be adjacent or at a distance from such a site. Several theories have been proposed to explain the "referred pain" phenomenon, with the convergence-projection theory the most widespread [16, 17]. Input from different tissue types converge on the same dorsal horn neurons [18]; After activation, increased nociceptive input is transmitted supraspinally and misinterpreted at the cortical level as pain from other tissues. It is possible that the reduction of space between the vertebrae as a consequence of the degenerative disk leads to increased pressure on spinal ligaments and other supporting tissues. This can be misinterpreted at the cortical level as pain from other tissues, like the hip region. Experimental studies have confirmed that noxious stimulation of interspinous ligament, facet joint, and paravertebral muscles causes referred pain that can radiate into the extremity [19-21].

Another explanation for the radiating pain from the higher lumbar spine can be found in the dermatomal innervations of the hip region. It is suggested that impingement of the higher lumbar spinal nerve roots (L1-L3) can cause pain in the dermatomal distribution surrounding the hip. The dermatomal distribution of the L1 spinal nerve is located in the groin and the upper part of the buttock. The distribution of the L2 spinal nerve is located in the outside thigh. It is possible that reduction of space between the vertebrae as a consequence of the degenerative disk is more likely to lead to impingement of the L1 and L2 nerve roots, and, therefore, causes pain in the dermatomal distribution. Spinal nerve roots pass through the intervertebral foramen as they travel from the spinal cord toward the periphery. It has been reported that narrowing of the disk space can reduce the vertical diameter of this intervertebral foramen [22].

The explanation for the stronger association between hip pain and disk space narrowing compared with the presence of osteophytes is unknown. This study evaluates the severity of anterior osteophytes, unfortunately we could not evaluate any bony aspects of the intervertebral foramen. The explanation for the stronger association between hip pain 
Table 2 Association between disk space narrowing and hip pain

Adjusted for age, gender, BMI, hip arthrosis, and low back pain

$* p \leq 0.05$

$* * p \leq 0.01$

OR odds ratio

CI Confidence interval

\begin{tabular}{llll}
\hline Narrowing level & N $(\%)$ & $\begin{array}{l}\text { Hip pain } \\
\text { OR }(95 \% \text { CI })\end{array}$ & $\begin{array}{l}\text { Chronic hip pain } \\
\text { OR }(95 \% \text { CI })\end{array}$ \\
\hline Men, $N=1,204$ & & \\
L1-L2 & $107(8.9)$ & $2.0(1.1-3.8)^{*}$ & $2.5(1.3-5.0)^{* *}$ \\
L2-L3 & $135(11.3)$ & $0.9(0.4-1.8)$ & $1.1(0.5-2.4)$ \\
L3-L4 & $153(12.7)$ & $1.1(0.6-2.1)$ & $1.1(0.5-2.2)$ \\
L4-L5 & $268(22.2)$ & $1.2(0.7-2.0)$ & $1.4(0.8-2.5)$ \\
L5-S1 & $408(33.9)$ & $0.7(0.4-1.1)$ & $0.6(0.4-1.1)$ \\
Women, $N=1,615$ & & & $1.8(1.1-2.7)^{* *}$ \\
L1-L2 & $201(12.5)$ & $1.7(1.1-2.5)^{*}$ & $1.6(1.1-2.3)^{*}$ \\
L2-L3 & $307(19.0)$ & $1.6(1.1-2.2)^{*}$ & $1.1(0.7-1.5)$ \\
L3-L4 & $342(21.1)$ & $0.9(0.7-1.3)$ & $1.0(0.7-1.4)$ \\
L4-L5 & $526(32.6)$ & $1.0(0.7-1.3)$ & $0.9(0.7-1.2)$ \\
L5-S1 & $662(41.0)$ & & $2.0(1.4-2.8)^{* *}$ \\
All, $N=2,819$ & & $1.8(1.3-2.5)^{* *}$ & $1.5(1.1-2.1)^{*}$ \\
L1-L2 & $308(10.9)$ & $1.4(1.0-1.9)^{*}$ & $1.1(0.8-1.5)$ \\
L2-L3 & $442(15.7)$ & $1.1(0.8-1.4)$ & $1.1(0.8-1.5)$ \\
L3-L4 & $495(17.6)$ & $1.0(0.8-1.3)$ & $0.8(0.6-1.1)$ \\
L4-L5 & $794(28.2)$ & $0.9(0.7-1.1)$ &
\end{tabular}

and disk space narrowing at L1/L2 in men compared with the association in women is also unknown. It is possible that even though women reported hip pain more often, only a small proportion of the complaints are due to disk space narrowing, whereas other factors determine the feeling of pain. Men and women could also report pain differently, therefore, effecting the association between hip pain, disk space narrowing, and gender. Cecchi et al. [23] showed that women presented with significantly more severe pain than men. Finally, the explanation for the absence of an association between hip pain and disk space narrowing at L2/L3 in men compared to women is also unknown. It is maybe due to an evidently lower prevalence of disk space narrowing at L2/ L3 in men compared to women.

Our study had several advantages. It was population based with a relatively high number of subjects. We used a semi-quantitative score, using standard radiographs, to characterize the presence and severity of hip and spine osteoarthritis. Assessment of the radiographs was carried out without knowledge of the questionnaire data, and so any errors in classification are likely to have been nondirectional. We defined chronic hip pain and chronic low back pain to be present if the duration of the hip joint pain was more than 1 year. In literature, others have chosen 3 months or even 6 months as the dividing line between acute and chronic pain [24]. However, with our definition, chronic pain included long lasting chronic complaints with long lasting impact on ones life.

However, there are several limitations in our explorative study that need to be considered when interpreting the results. Our data did not include the precise location of the hip pain. This limitation is partly undermined by the fact that the dermatomal distribution of L1 and L2 includes the upper part of the buttock, the groin, and the lateral thigh, which cover a wide area of the hip region. Further, our data did not include a clinical evaluation of the hip pain. In this way we could not account for the potential of soft-tissue pathology contributing to the reported hip pain.

Moreover, hip osteoarthritis was only considered when the Kellgren \& Lawrence score of one or both joints was equal to or larger than two in agreement with conventional epidemiological definitions for hip osteoarthritis [25]. In this way there is still a possibility of the presence of hip osteoarthritis which is not clearly visible yet on radiographs at that time point. In order to exclude the possibility of this confounding, we reanalyzed the data with adjusting for the presence of radiographic hip osteoarthritis 6.6 years later. We defined a new variable that included all the participants with hip osteoarthritis at baseline and/or hip osteoarthritis 6.6 years later $(n=413)$. The strength of the associations was unchanged (for chronic pain the L1/L2 OR was 1.9; $95 \%$ CI 1.3-2.7; again higher in men $(\mathrm{OR}=2.7 ; 95 \%$ CI $1.4-5.3)$ than in women $(\mathrm{OR}=1.7$; $95 \%$ CI 1.1-2.6).

Furthermore, there could be some selection bias in favor of relatively healthy participants. The participants in the present study had to be mobile enough to visit the research center for X-ray examination, both for the baseline and follow-up appointments (mean 6.6 years) [9]. In other words, patients with the most severe symptoms were most 
likely not included, but this may be inevitable in long-term prospective cohort studies.

What are the implications of these findings for researchers and clinicians?

Accurate diagnosis of pain originating from the hip joint can be clinically challenging. There have been several studies about the usefulness of hip injections to differentiate intra-articular causes of hip pain from spinal causes [4-7]. To our knowledge, there have been no studies about the usefulness of local spine infiltrations to differentiate hip and spine originated hip pain. A possible explanation for this is the availability of a successful treatment for degenerated hip disease (hip arthroplasty), but less predictable treatment options for degenerative spine disorders.

The differentiation of signs and symptoms suggestive of a hip disorder is important in giving patients adequate information regarding their condition and for applying the most beneficial treatment. Our data provides evidence for an association between hip pain and disk space narrowing at disk level L1/L2 and L2/L3. In case of uncertainty of the cause of hip pain, evaluation of lumbar radiographs may help to identify those hip pain patients who might have pain arising from the lumbar spine. Perhaps hip infiltration in patients without higher lumbar disk degeneration is even unnecessary. However, well-designed studies are needed to verify this hypothesis.

\section{Conclusion}

In conclusion, this study explores the association of selfreported hip pain with lumbar spine osteoarthritis. Our data provide evidence for an association between hip pain and disk space narrowing at disk level L1/L2 and L2/L3. In case of uncertainty of the cause of hip pain, evaluation of lumbar radiographs may help to identify those hip pain patients who might have pain arising from the lumbar spine. Welldesigned studies are needed to verify this hypothesis.

Acknowledgments No financial or material support for the research and the work was received.

\section{References}

1. Christmas C, Crespo CJ, Franckowiak SC et al (2002) How common is hip pain among older adults? Results from the Third National Health and Nutrition Examination Survey. J Fam Pract 51(4):345-348

2. Illgren R, Honkamp N, Weisman M et al (2006) The diagnostic and predictive value of hip anesthetic arthrograms in selected patients before total hip arthroplasty. J Arthroplast 21(5): 724-730
3. Brown MD, Gomez-Marin O, Brookfield KF et al (2004) Differential diagnosis of hip disease versus spine disease. Clin Orthop Relat Res 419:280-284

4. Ashok N, Sivan M, Tafazal S (2009) The diagnostic value of anaesthetic hip injection in differentiating between hip and spinal pain. Eur J Orthop Surg Traumatol 19:167-171

5. Faraj A, Kumaraguru P, Kosygan K (2003) Intra-articular bupivacaine hip injection in differentiation of coxarthrosis from referred thigh pain: a 10 year study. Acta Orthop Belg 69(6):518-521

6. Kleiner J, Thorne R, Curd J (1991) The value of buvicaine hip injection in the differentiation of coxarthrosis from lower extrimity neuropathy. J Rheumatol 18(3):422-427

7. Pateder D, Hungerford M (2007) Use of fluoroscopically guided intra-articular hip injection in differentiating the pain source in concomitant hip and lumbar spine arthritis. Am J Orthop 36(11): 591-593

8. Hofman A, Breteler MM, van Duijn CM et al (2007) The Rotterdam study: objectives and design update. Eur J Epidemiol 22(11):819-829

9. Reijman M, Hazes JM, Pols HA et al (2005) Role of radiography in predicting progression of osteoarthritis of the hip: prospective cohort study. BMJ 330(7501):1183

10. Kettler A, Wilke HJ (2006) Review of existing grading systems for cervical or lumbar disc and facet joint degeneration. Eur Spine J 15(6):705-718

11. Lane NE, Nevitt MC, Genant HK et al (1993) Reliability of new indices of radiographic osteoarthritis of the hand and hip and lumbar disc degeneration. J Rheumatol 20(11):1911-1918

12. de Schepper EI, Damen J, van Meurs JB et al (2010) The association between lumbar disc degeneration and low back pain: the influence of age, gender and individual radiographic features. Spine 35(5):531-536

13. Lievense AM, Bierma-Zeinstra SM, Verhagen AP et al (2002) Influence of obesity on the development of osteoarthritis of the hip: a systematic review. Rheumatology (Oxford) 41(10):1155-1162

14. Pye SR, Reid DM, Adams JE et al (2007) Influence of weight, body mass index and lifestyle factors on radiographic features of lumbar disc degeneration. Ann Rheum Dis 66(3):426-427

15. Tukker A, Visscher T, Picavet H (2009) Overweight and health problems of the lower extremities: osteoarthritis, pain and disability. Public Health Nutr 12(3):359-368

16. Frey Law LA, Sluka KA, McMullen T et al (2008) Acidic buffer induced muscle pain evokes referred pain and mechanical hyperalgesia in humans. Pain 140(2):254-264

17. Graven-Nielsen T (2006) Fundamentals of muscle pain, referred pain, and deep tissue hyperalgesia. Scand J Rheumatol 35:1-43

18. Willis WD, Coggeshall RE (2004) Sensory mechanisms of the spinal cord. Kluwer Academic/Plenum Publishers, New York

19. Feinstein R, Langton J, Jameson R et al (1954) Experiments on pain referred from deep somatic tissues. J Bone Jt Surg Am 36A:981-997

20. Kellgren J (1939) On the distribution of pain arising from deep somatic structures with charts of segmental pain areas. Clin Sci 4:35-46

21. O'Neill CW, Kurgansky ME, Derby R et al (2002) Disc stimulation and patterns of referred pain. Spine 27:2776-2781

22. Cinotti G, De Santis P, Nofroni I et al (2002) Stenosis of lumbar intervertebral foramen. Spine 27(3):223-229

23. Cecchi F, Debolini P, Lova RM et al (2006) Epidemiology of back pain in a representative cohort of Italian persons 65 years of age and older: the InCHIANTI study. Spine 31(10):1149-1155

24. Bonica J (1990) The management of pain. Lea and Febiger, Philadelphia

25. Reijman M, Hazes JM, Pols HA et al (2004) Validity and reliability of three definitions of hip osteoarthritis: cross sectional and longitudinal approach. Ann Rheum Dis 63(11):1427-1433 